Andrea Giampreti*, Georgios Eleftheriou, Mariapina Gallo, Raffaella Butera, Gioia Contessa, Lorella Faraoni, Anna Sangiovanni, Giulia Negri, Giovanna Falchi and Giuseppe Bacis

\title{
Medications prescriptions in COVID-19 pregnant and lactating women: the Bergamo Teratology Information Service experience during COVID-19 outbreak in Italy
}

https://doi.org/10.1515/jpm-2020-0339

Received July 17, 2020; accepted September 27, 2020; published online October 15, 2020

\section{Abstract}

Objectives: The severe acute respiratory syndrome coronavirus 2 (COVID-19) outbreak in Italy, especially in Lombardy and Bergamo city, represented probably nowadays one of the first major clusters of COVID-19 in the world. The aim of this report is to describe the activity of Bergamo Teratology Information Service (TIS) in supporting the public and health-care personnel in case of drug prescriptions in suspected/confirmed COVID-19 pregnant and lactating patients during COVID-19 outbreak in Italy.

Methods: All Bergamo TIS requests concerning COVID-19 pregnant and lactating women have been retrospectively evaluated from 1 March to 15 April 2020. Type of medications, drug's safety profile and compatibility with pregnancy and lactation are reported.

Results: Our service received information calls concerning 48 (9 pregnant, 35 lactating) patients. Among pregnant and lactating women, the requests of information were related to 16 and 60 drugs prescriptions respectively. More than half concerned drugs prescriptions during the first and second trimester (13/16) and during the first six months of lactation (37/60). Hydroxychloroquine and azithromycin were the most involved.

Conclusions: Hydroxychloroquine and azithromycin at dosages used for COVID-19 may be considered compatible and reasonably safe either in pregnancy and lactation. Antivirals may be considered acceptable in pregnancy.

*Corresponding author: Dr. Andrea Giampreti (Medical Doctor Physician), Bergamo Teratology Information Service, Papa Giovanni XXIII Hospital, 24100 Bergamo Italy, Phone: +39 035 2674460, Fax: +39035 2674835, E-mail: agiampreti@asst-pg23.it

Georgios Eleftheriou, Mariapina Gallo, Raffaella Butera, Gioia Contessa, Lorella Faraoni, Anna Sangiovanni, Giulia Negri, Giovanna Falchi and Giuseppe Bacis, Bergamo Teratology Information Service, Papa Giovanni XXIII Hospital, 24100 Bergamo Italy
During lactation lopinavir and ritonavir probably exhibit some supportive data from literature that darunavir and cobicistat do not. Tocilizumab may be considered for COVID-19 treatment because no increased malformation rate were observed until now. However caution may be advised because human data are limited and the potential risk of embryo-fetal toxicity cannot be excluded.

Keywords: COVID-19; lactation; pregnancy; Teratology; toxicology.

\section{Introduction}

The severe acute respiratory syndrome coronavirus 2 (SARS-CoV-2, COVID-19) outbreak is challenging the Italian health care system with a critical impact on all the health activities $[1,2]$ especially in patients' management, including drug therapy administration in suspected or confirmed COVID-19 pregnant and lactating patients. Many researchers are evaluating the effectiveness and safety of several drugs for SARS-CoV-2 infection as antiviral pro-drugs [3] and interleukin-6 receptor antagonists [4] and, currently the pharmacological approach to treat COVID-19 patients may involve different drugs ranging from hydroxychloroquine [5] to lopinavir/ritonavir [6] associated to other supportive and symptomatic therapies and antibacterial drugs, such as azithromycin, cefixime or ceftriaxone. The aim of this report is to describe the activity of Bergamo Teratology Information Service (TIS) in supporting the public and health care personnel in case of drug prescriptions in suspected or confirmed COVID-19 pregnant and lactating patients during COVID-19 outbreak in Italy.

\section{Materials and methods}

Bergamo Poison Control Center and Teratology Information Service (TIS) is a 24-h service, operating at Papa Giovanni XXIII Hospital since 
1 January 2000, that provides information to the public and health care personnel on poisoning exposures and on pharmacotherapy during pregnancy and breastfeeding. All Bergamo TIS requests concerning suspected or confirmed COVID-19 pregnant and lactating women have been retrospectively evaluated during COVID-19 outbreak in Italy, from 1 March to 15 April 2020. Type of medications involved, drug's safety profile and compatibility with pregnancy and lactation are reported. Moreover also demographical data as age, gestational age and time of lactation are reported. A suspected COVID-19 patient was considered a patient with symptoms compatible with SARS-CoV-2 infection or in close contact with a confirmed COVID-19 patient. Patients were considered COVID-19 confirmed cases when reported positive nasopharyngeal swab or confirmed diagnosis for COVID-19. Informed consent was obtained from all individuals included in this study.

The research has been complied with all the relevant national regulations, institutional policies and in accordance the tenets of the Helsinki Declaration, and has been approved by the Ethical Committee approval.

\section{Results}

During the study period ranging from 1 March to 15 April 2020, our service received information calls concerning 48 patients with suspected $(20 / 48 ; 41 \%)$ or confirmed (28/48; 59\%) COVID-19. Among these, 44 involved requests for medications in COVID-19 pregnant and lactating women (Figure 1): nine pregnant patients (2 suspected and 7 confirmed COVID-19) and 35 lactating patients (14 suspected and 21 confirmed COVID-19). Main calls came from Lombardy region (26) and Emilia Romagna (6). The other regions asked for less requests (Veneto 3, Piemonte 3, Marche 2, Campania, Liguria, Toscana and Sicilia 1 each). Bergamo TIS has been contacted by 16 physicians (7 pediatricians, five gynecologists, two emergency physicians, 1 infective disease specialist, 1 psychiatrist), 27 patients and 1 pharmacist (Figure 1). The mean age of the patients was 33 years old (20-47), 29 years for pregnant (20-36) and 35 years for lactating women (26-47).
Among pregnant women, the requests of information were related to 16 drug prescriptions (Figure 2); seven during first trimester, six during second trimester, three during third trimester. Type of medications involved were: hydroxychloroquine (4), azithromycin (3), acetaminophen (2), lopinavir/ritonavir (1), tocilizumab (1), clarithromycin (1), amoxicillin/clavulanate (1), piperacillin (1), meropenem (1), dextromethorphan (1). All prescriptions were considered safe for pregnant patients. Among those that received hydroxychloroquine during the first trimester and for the patient that received lopinavir/ritonavir, despite an increased teratogenic risk has not been expected, a precautionary second level ultrasound at 20-week was indicated. Tocilizumab information were requested for a compassionate administration in a 20 years old pregnant 20-week patient with a confirmed severe COVID-19. The prescription was considered compatible also taking in account the severity of the clinical picture and the risk-benefit ratio for the patient. The remaining drugs (antibiotics, acetaminophen and dextromethorphan) were considered reasonably safe.

Among lactating women, Bergamo TIS received information requests for 60 drug prescriptions (Figure 3); 16 during the first month ( 6 during colostrum), 21 during the 1-6 months period and 23 over the six months period. Type of medications involved were: hydroxychloroquine (21), azithromycin (7), darunavir (5), ibuprofen (5), lopinavir/ ritonavir (4), levofloxacin (4), ketoprofen (2), amoxicillin/ clavulanate (2), remdesivir (1), cobicistat (1), pidotimod (1), acetaminophen (1), phopshomycin (1), acetylcysteine (1), heparin (1), lorazepam (1), betamethasone (1), acetylsalicylate $100 \mathrm{mg} /$ die (1). Among the most involved drugs hydroxychloroquine and azithromycin, both have been considered compatible and safe due to their estimated low breast milk concentrations. Concerning antivirals, lopinavir and ritonavir have not evidenced adverse events in breastfed infants from mother on therapy, so they have

\begin{tabular}{lccc} 
& $\begin{array}{c}\text { Consulting } \\
\text { requested for } \\
\text { compatibility in } \\
\text { PREGNANCY }\end{array}$ & $\begin{array}{c}\text { Consulting } \\
\text { requested for } \\
\text { compatibility in } \\
\text { LACTATION }\end{array}$ & $\begin{array}{c}\text { Total } \\
\text { (n. patients) }\end{array}$ \\
\hline $\begin{array}{l}\text { Consulting } \\
\text { requested by }\end{array}$ & 6 & 10 & 16 \\
\hline Physicians & - & 1 & 1 \\
\hline Pharmacist & 3 & 24 & 27 \\
\hline Patients & 9 & 35 & 44 \\
\hline Total & & & \\
\hline
\end{tabular}

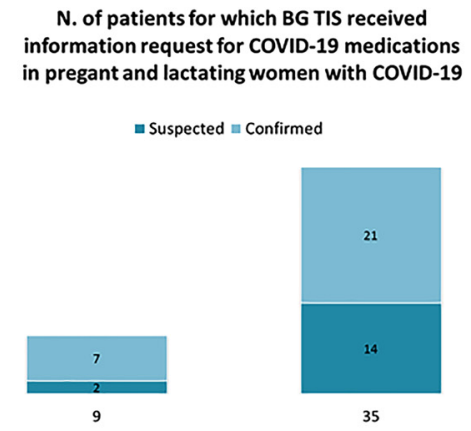

Pregnant COVID-19 patients

Figure 1: Bergamo TIS consulting for medications in COVID-19 pregnant and lactating patients. 


\begin{tabular}{lcccc} 
& $\mathbf{1}^{\text {st }}$ trimester & $\mathbf{2}^{\text {nd }}$ trimester & $\mathbf{3}^{\text {rd }}$ trimester & Total \\
\hline Medications & & & \\
\hline Hydroxychloroquine & 3 & 1 & 1 & 4 \\
Azithromycin & 2 & 1 & 1 & 3 \\
Acetaminophen & 1 & 1 & & 1 \\
\hline $\begin{array}{l}\text { Lopinavir/Ritonavir } \\
\text { Tocilizumab }\end{array}$ & 1 & 1 & & 1 \\
Clarithromycin & & 1 & & 1 \\
Amoxicillin/Clavulanate & & 1 & 1 & 1 \\
$\begin{array}{l}\text { Piperacillin } \\
\text { Meropenem }\end{array}$ & & & $\mathbf{3}$ & 1 \\
Dextromethorphan & & $\mathbf{6}$ & & 1 \\
\hline Total & $\mathbf{7}$ & &
\end{tabular}

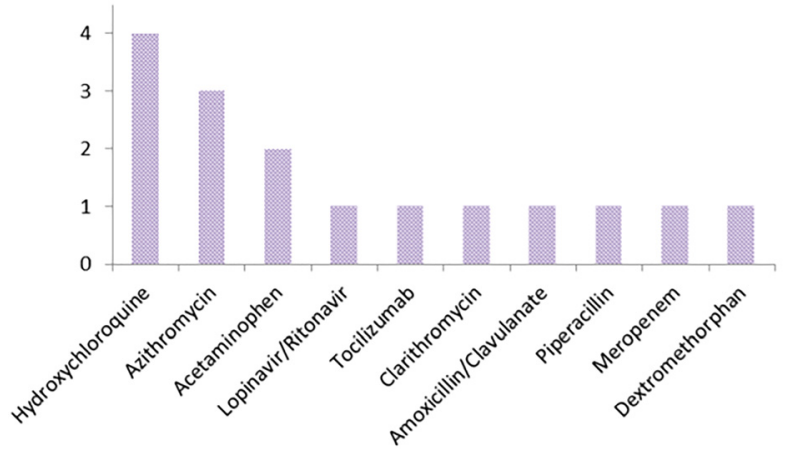

Figure 2: Bergamo TIS consulting for medications in COVID-19 pregnant patients.

been considered compatible in lactation. On the other hand, despite no published data are available on their use during breastfeeding, darunavir and cobicistat were considered compatible with breastfeeding, according to their kinetic parameters these drugs could be minimally excreted in breast milk. Remdesivir was requested for the therapy of a mother that had suspended medicine $24 \mathrm{~h}$ before TIS consulting; considering the absence of specific kinetic and clinical data, the mother was advised to wait $72 \mathrm{~h}$ after drug interruption (about 3 half-lives) before restarting lactation. About pidotimod no data are available for its use in lactation; so we consider to avoid the drug during breastfeeding. Among the remaining drugs, antibiotics (amoxicillin/clavulanate, levofloxacin, phopshomycin), anti-inflammatory drugs (ketoprofene, betamethasone), acetaminophen, acetylcysteine, low dose acetylsalicylate, heparin and lorazepam were considered reasonably safe and compatible, as in other conditions, regardless of COVID-19. Women in colostrum phase ( $<3$ days) were 4 (3 confirmed and 1 suspected COVID-19) and during the consultation were asked for delivery and infant condition at birth: among these no malformation or birth defects were reported, one delivered at 27 week and healthy pre-term infant. Finally, a phone-call follow-up among lactating women was performed and 18/35 patients answered: 12 reported no adverse effects during breastfeeding, four reported lactation interruption and two do not assumed medications for which information were requested. Among women who reported no adverse effects during breastfeeding and were able to remember dosage regimen and therapy duration, three received hydroxychloroquine $800 \mathrm{mg}$ once followed by $400 \mathrm{mg}$ daily for 2 weeks, three received azithromycin $500 \mathrm{mg}$ daily for one week and one woman was treated with darunavir $1200 \mathrm{mg} /$ ritonavir $200 \mathrm{mg}$ daily for 5 days.

\section{Discussion}

On February 20, 2020, a young man in Lombardy region of North Italy was admitted with an atypical pneumonia that later proved to be COVID-19; this was the beginning of one of the largest and most serious clusters of COVID-19 in the world [7]. In our experience, most patients that directly contacted us or for which our service has been consulted

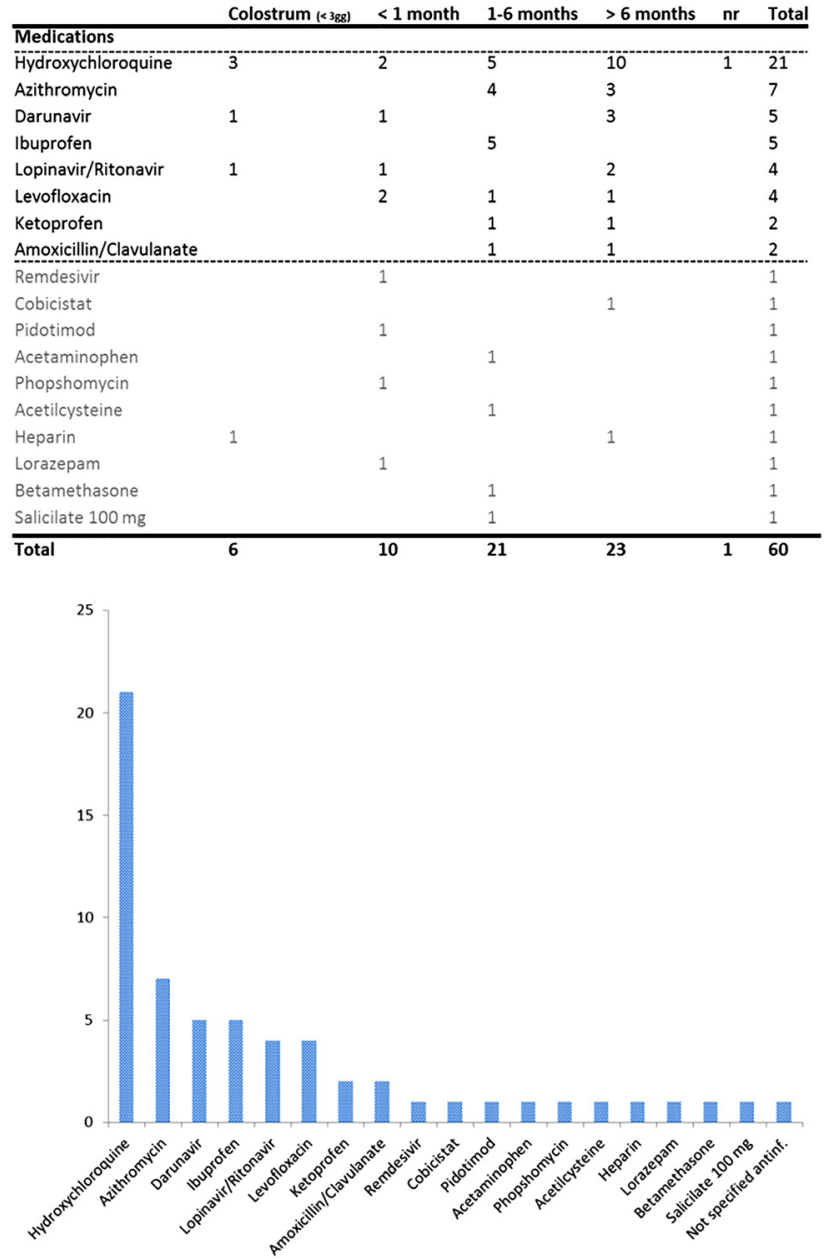

Figure 3: Bergamo TIS consulting for medications in COVID-19 lactating patients. 
for $(28 / 44 ; 63 \%)$ were confirmed COVID-19 cases and most requests involved safety and compatibility of hydroxychloroquine and azithromycin both in pregnancy (mainly first and second trimester) and lactation (mainly over 1 month).

\section{Medications prescriptions in COVID-19 pregnant women}

\section{Hydroxychloroquine}

In recent in vitro studies, hydroxychloroquine appears to inhibit SARS-CoV-2 viral replication and has the potential to interfere with other virus host interactions such as its fusion with the cell membrane, post-translational modification and viral release [5, 8]. As this is a clinically available drug, it is likely that in many cases of pregnant women contracting the virus, the issue of fetal safety of chloroquine will emerge [9]. The ototoxic and retino-toxic findings in infants who were exposed to chloroquine in utero and similar findings in some animal studies raised some concerns about the safety of chloroquine and hydroxychloroquine use during pregnancy $[10,11,12]$. To date, the most recent systematic review and meta-analysis regarding hydroxychloroquine use during pregnancy
(200-400 mg/day from 3 months before and throughout pregnancy) detected no significant increased risk of major malformations in general, as well as craniofacial, cardiovascular, nervous system and genitourinary malformations in infants. Additionally, no significant increase in the rates of stillbirth, low birth weight and prematurity were detected [10] (Table 1).

\section{Macrolids: azithromycin and clarithromycin}

Azithromycin is well studied in pregnancy and its use for COVID-19 pregnant patients do not seem to be different in term of fetal risks from its use in other conditions. Experimental animal studies and human reports show that azithromycin use during pregnancy is not expected to increase the risk of birth defects [13]. Clarithromycin on the other hand may produce adverse pregnancy outcome (e.g. possible risk of cardiovascular anomalies, spontaneous abortion and IUGR) in experimental animals at loworder multiples of the human dose level on a $\mathrm{mg} / \mathrm{kg}$ basis [14]. A potential increased hazard of miscarriage but no increased prevalance of having offspring with malformations among women redeeming a prescription of clarithromycin in early pregnancy has been recently described [15]. However different human data do not

Table 1: Safety in pregnancy and compatibility in lactation of drugs prescriptions in COVID-19 patients.

\begin{tabular}{|c|c|c|}
\hline Drugs prescriptions in COVID-19 patients & Pregnancy & Lactation \\
\hline Acetaminophen & Safe & Compatible \\
\hline Acetylsalicilate (low dose) & Safe & Compatible \\
\hline Acetylcysteine & Safe & Compatible \\
\hline Amoxicillin/Clavulanate* & Safe (cautions in prenatal phase) & Compatible \\
\hline Azithromycin & Safe & Compatible \\
\hline Betamethasone & Safe & Compatible \\
\hline Clarithromycin & Probably safe & Compatible \\
\hline Cobicistat & Safe & No data, probably compatible \\
\hline Darunavir & Probably safe & No data, probably compatible \\
\hline Dextromethorphan & Safe & Compatible \\
\hline Hydroxychloroquine & Safe & Compatible \\
\hline Heparin / LW Heparins & Safe & Compatible \\
\hline Ibuprofen & Safe (until 30 weeks) & Compatible \\
\hline Ketoprofen & Safe (until 30 weeks) & Compatible \\
\hline Lorazepam & Safe & Compatible \\
\hline Levofloxacin & No first choice & Compatible \\
\hline Lopinavir/ritonavir & Safe & Compatible \\
\hline Meropenem & Probably safe (caution for lack of systematic investigations) & Compatible \\
\hline Phopshomycin & Safe & Compatible \\
\hline Pidotimod & No data & No data \\
\hline Piperacillin/Tazobactam & Safe & Compatible \\
\hline Remdesivir & No data & No data \\
\hline Tocilizumab & Probably safe (caution for limited human data) & Compatible \\
\hline
\end{tabular}


underline an increase of fetal risks malformations during clarithromycin therapy in pregnancy $[16,17]$ and make the use of this drug probably safe in pregnancy. Alternative antibiotics, with the exception of clinical circumstances where no other therapies are appropriate, could be considered during pregnancy.

\section{Amoxicillin/clavulanate}

Few studies have discussed an association with cleft palate and use of amoxicillin [18] with an absolute cleft risk of 2-4 per 1,000, a modest increase compared to the background risk. Other authors could not find an increased risk for oral clefts after intrauterine amoxicillin exposure [19]. Moreover a large population wide cohort study found, with a high degree of precision, no increased risk of congenital malformations following first-trimester exposure to broadspectrum penicillins as amoxicillin [20]. In a large, randomized multicenter trial, the prenatal use of ampicillin and clavulanic acid was associated with an increase in the occurrence of neonatal necrotizing enterocolitis [21] but other studies do not confirm this concern [22]. Avoiding, if possible, clavulanate in the prenatal part of pregnancy may be prudent.

\section{Tocilizumab}

Even if requested for only one case, a peculiar consideration need tocilizumab, an interleukin- 6 receptor inhibitor, administered for the treatment of rheumatoid arthritis, active systemic juvenile idiopathic arthritis and polyarticular juvenile idiopathic arthritis that nowadays is under investigation for treatment of COVID-19 infection. Concerning tocilizumab, despite limited human pregnancy reports and an estimated malformation rate up to $4.5 \%$ [23], several authors report no increased malformation rate after tocilizumab administration in pregnancy $[24,25,26]$. However it is known that tocilizumab may actively crosses the placenta in late pregnancy and may affect the fetal immune response. In animal reproduction studies, intravenous administration of tocilizumab during organogenesis in cynomolgus monkeys resulted in miscarriage or stillbirth. Nowadays it is not yet possible to predict drugrelated birth defects and the risk of miscarriage [27, 28]. Caution should be advised during pregnancy because of inadequate human data and the possible risk of embryofetal toxicity. In our case the prescription was considered compatible according to the severity of the clinical picture and the risk-benefit ratio for the patient.

\section{Lopinavir and ritonavir}

Concerning anti-retroviral therapy the reported experience with human pregnancy exposure to these medications is limited. Ritonavir in rats, resulted in an increase in resorptions, a decrease in fetal weight, and a delayed ossification at a dose level producing maternal toxicity, which resulted in exposures $30 \%$ of those achieved in human therapy [29]. Human data derived from the Antiretroviral Pregnancy Registry showed that the prevalence of birth defects among infants prenatally exposed to lopinavir/ritonavir is not significantly different from internal or external comparison groups. These data provide reassuring information to patients and clinicians about the safety of lopinavir and ritonavir [30]. Avoiding oral solution during pregnancy due to alcohol and propylene glycol content may be advised if possible.

\section{Meropenem}

Both carbapenems and monobactams cross the placenta and may reach the fetus in relevant quantities. Based on experimental animal studies, meropenem therapy during pregnancy is not expected to increase the risk of congenital malformations. There were no adverse effects in the offspring after treatment with up to 1.8 (rats) and 3.7 times the human dose [31]. However, systematic investigations have not been conducted and meropenem may be used when resistance testing indicates that they are needed.

\section{Medications prescriptions in COVID-19 lactating women}

In lactating women most requests were related to therapy with hydroxychloroquine, azithromycin, lopinavir, ritonavir and darunavir.

\section{Hydroxychloroquine}

A review of the cases regarding the milk sample concentrations of more than 50 women who used hydroxychloroquine during breastfeeding (100-400 mg/day) suggested a relative infant dose (RID) between 1.9 and 3.2\% [32] and the available evidence regarding lactation also suggests that hydroxychloroquine is compatible with breastfeeding [33] because of breastfed infants receive only small amounts of the drug in breastmilk. In infants up to at least 1 year of age, careful follow-up found no adverse effects on growth, vision or hearing (Table 1). 


\section{Azithromycin}

Azithromycin would not be expected to cause adverse effects in breastfed infants because of the low levels in breastmilk. As for other antibiotic therapies in lactating mother monitoring the infant for possible effects on the gastrointestinal flora, such as vomiting, diarrhea, candidiasis (thrush, diaper rash) could be indicated.

\section{Lopinavir and ritonavir}

Lopinavir has been well studied during breastfeeding. Among nine breastfed infants whose mothers were taking lopinavir $400 \mathrm{mg}$ with ritonavir $100 \mathrm{mg}$ twice daily as part of a multi-drug treatment for HIV infection, no adverse effects were observed by investigators or reported by mothers at 1, 3 and 6 months of age [34]. The infants' median lopinavir plasma concentrations has been detected in $105 \mu \mathrm{g} / \mathrm{L}$ (range $12-518 \mu \mathrm{g} / \mathrm{L}$ ), which is a median of $8 \%$ (range $0-16 \%$ ) of the maternal serum concentration. This consideration set lopinavir among acceptable drugs in lactation [35]. Concerning ritonavir, 30 nursing mothers were studied at 6,12 or 24 weeks postpartum (10 at each time). Each mother was taking ritonavir $100 \mathrm{mg}$ twice daily by mouth starting at delivery. Infant plasma samples were obtained before their mother's first dose and at 2, 4 and $6 \mathrm{~h}$ after the mother's dose. Infants were allowed to breastfeed ad libitum during the study period. Ritonavir was undetectable $(<10 \mu \mathrm{g} /$ L) in all of the 115 infant plasma samples [36].

\section{Darunavir and cobicistat}

Darunavir and cobicistat are not investigated in lactating women; however their kinetic parameters (e.g. protein binding $>95 \%$, molecular weight $>500 \mathrm{da}$, and low oral bioavailability $37 \%$ for darunavir) make these antivirals potentially compatible with breastfeeding (Table 1).

\section{Conclusions}

COVID-19 outbreak in Italy, especially in Lombardy and our province of Bergamo, represents probably nowadays one of the first major clusters of COVID-19 in the world. During this dramatic outbreak, Bergamo TIS continued its $24 \mathrm{~h}$ consulting activity as only one physician of our staff (as from other departments) was involved in the direct management of COVID-19 patients. Data from our experience showed that, as for in other pathological conditions, the administration of hydroxychloroquine and azithromycin at dosages used for COVID-19 may be considered compatibleand reasonably safe either in pregnancy (throughout the pregnancy) and during lactation. Antivirals may be considered compatible in pregnant and lactating patients with COVID-19. Lopinavir and ritonavir probably exhibit some supportive data from literature that darunavir and cobicistat do not, particularly for pregnancy. In absence of alternative drugs these latter could be considered in lactation due to their kinetic parameters that make them theoretically minimally excreted in the breast milk. Tocilizumab may be considered for COVID-19 treatment because no increased malformation rate were observed until now and with low risk-benefit ratio for the patient. However caution may be advised because human data are limited and the potential risk of embryo-fetal toxicity cannot be excluded. The other medications, with the exception of pidotimod, resulted reasonably safe for pregnant and compatible for lactating women as for in other pathological conditions. It should be kept in mind that safety of medications in pregnancy and lactation is an ever changing topic. When there are conflicting and debated data healthcare providers should present patients with the most current and compelling evidence from literature. Balancing potential benefits, potential risks and alternatives is the most challenging task aimed the patients to make an informed decision. Bergamo TIS provided an essential support to those COVID-19 patients needing reassurance and health care personnel that need to evaluate therapies' safety profile in COVID-19 pregnant and lactating patients.

Research funding: None declared.

Author contributions: All authors have accepted responsibility for the entire content of this manuscript and approved its submission.

Competing interests: Authors state no conflict of interest. Informed consent: Informed consent was obtained from all individuals included in this study.

Ethical approval: The research has been complied with all the relevant national regulations, institutional policies and in accordance the tenets of the Helsinki Declaration, and has been approved by the Ethical Committee approval.

\section{References}

1. Bernucci C, Brembilla C, Veiceschi P. Effects of the COVID-19 outbreak in northern Italy: perspectives from the Bergamo neurosurgery department. World Neurosurg 2020;137:456-8.e1.

2. Perin A, Servadei F, DiMeco F. 'Hub and Spoke' Lombardy neurosurgery group. May we deliver neuro-oncology in difficult times (e.g. COVID-19)? J Neuro Oncol 2020;148:203-5. 
3. Grein J, Ohmagari N, Shin D, Diaz D, Asperges E, Castagna A, et al. Compassionate use of remdesivir for patients with severe COVID19. N Engl J Med 2020;382:2327-36.

4. Zhang W, Zhao Y, Zhang F, Wang Q, Taisheng L, Zhengyin L, et al. The use of anti-inflammatory drugs in the treatment of people with severe coronavirus disease 2019 (COVID-19): the perspectives of clinical immunologists from China. Clin Immunol 2020;214:108393.

5. Yao X, Ye F, Zhang M, Cui C, Huang B, Niu P, et al. In vitro antiviral activity and projection of optimized dosing design of hydroxychloroquine for the treatment of severe acute respiratory syndrome coronavirus 2 (SARS-CoV-2). Clin Infect Dis 2020;71:732-9.

6. Ye XT, Luo YL, Xia SC, Sun QF, Ding JG, Zhou Y, et al. Clinical efficacy of lopinavir/ritonavir in the treatment of Coronavirus disease 2019. Eur Rev Med Pharmacol Sci 2020;24:3390-6.

7. Livingston E, Bucher K. Coronavirus disease 2019 (COVID-19) in Italy. JAMA 2020;323:1335.

8. Devaux CA, Rolain JM, Colson P, Raoult D. New insights on the antiviral effects of chloroquine against coronavirus: what to expect for COVID-19? Int J Antimicrob Agents 2020:55:105938.

9. Kaplan YC, Koren G. Use of hydroxychloroquine during pregnancy and breastfeeding: an update for the recent coronavirus pandemic (COVID-19). Motherisk Int J 2020;1:9.

10. Kaplan YC, Ozsarfati J, Nickel C, Koren G. Reproductive outcomes following hydroxychloroquine use for autoimmune diseases: a systematic review and meta-analysis. Br J Clin Pharmacol 2016; 81:835-48.

11. Ullberg S, Lindquist NG, Sjostrand SE. Accumulation of chorioretinotoxic drugs in the foetal eye. Nature 1970;227: 1257-8.

12. Paufique L, Magnard P. Retinal degeneration in two children following preventive antimalarial treatment of the mother during pregnancy. Bull Soc Ophtalmol Fr 1969;69:466-7.

13. Sarkar M, Woodland C, Koren G, Einarson AR. Pregnancy outcome following gestational exposure to azithromycin. BMC Pregnancy Childbirth 2006;6:18.

14. Rao GN, Giles HD. Reproductive and developmental toxicity of AIDS combination therapies in Swiss (CD-1N) mice. Toxicologist 2000;54:227.

15. Andersen JT, Petersen M, Jimenez-Solem E, Broedbaek K, Andersen $\mathrm{NL}$, Torp-Pedersen C, et al. Clarithromycin in early pregnancy and the risk of miscarriage and malformation: a register based nationwide cohort study. PloS One 2013;8:e53327.

16. Bar-Oz B, Diav-Citrin O, Shechtman S, TellemR, ArnonJ, Franceticl, et al. Pregnancy outcome after gestational exposure to the new macrolides: a prospective multi-center observational study. Eur J Obstet Gynecol Reprod Biol 2008;141:31-4.

17. Bérard A, Sheehy 0 , Zhao JP, Nordeng $\mathrm{H}$. Use of macrolides during pregnancy and the risk of birth defects: a population-based study. Pharmacoepidemiol Drug Saf 2015;24:1241-8.

18. Lin KJ, Mitchell AA, Yau WP, Louik C, Hernández-Díaz S. Maternal exposure to amoxicillin and the risk of oral clefts. Epidemiology 2012;23:699-705.

19. Mølgaard-Nielsen D, Hviid A. Maternal use of antibiotics and the risk of orofacial clefts: a nationwide cohort study.

Pharmacoepidemiol Drug Saf 2012;21:246-53.

20. Damkier P, Brønniche LMS, Korch-Frandsen JFB, Broe A. In utero exposure to antibiotics and risk of congenital malformations: a population-based study. Am J Obstet Gynecol 2019;221:648.e1648.e15.
21. Kenyon SL, Taylor DJ, Tarnow-Mordi W. Broad-spectrum antibiotics for preterm, prelabour rupture of fetal membranes: the ORACLE I randomised trial. ORACLE collaborative group. Lancet 2001;357:979-88.

22. Ehsanipoor RM, Chung JH, Clock CA, McNultyJA, WingDA. A retrospective review of ampicillin-sulbactam and amoxicillin + clavulanate vs cefazolin/cephalexin and erythromycin in the setting of preterm premature rupture of membranes: maternal and neonatal outcomes. Am J Obstet Gynecol 2008;198:e54-6.

23. Hoeltzenbein M, Beck E, Rajwanshi R, Gotestam Skorpen C, Berber $E$, Schaefer $C$, et al. Tocilizumab use in pregnancy: analysis of a global safety database including data from clinical trials and postmarketing data. Semin Arthritis Rheum 2016;46:238-45.

24. Weber-Schoendorfer C, Schaefer C. Pregnancy outcome after tocilizumab therapy in early pregnancy-a case series from the German Embryotox Pharmacovigilance Center. Reprod Toxicol 2016;60:29-32. Epub 2016 Jan 21. PubMed PMID: 26806369.

25. Nakajima K, Watanabe O, Mochizuki M, Nakasone A, Ishizuka N, Murashima A. et al. Pregnancy outcomes after exposure to tocilizumab: a retrospective analysis of 61 patients in Japan. Mod Rheumatol 2016;26:667-71.

26. Kaneko K, Sugitani M, Goto M, Murashima A. Tocilizumab and pregnancy: four cases of pregnancy in young women with rheumatoid arthritis refractory to anti-TNF biologics with exposure to tocilizumab. Mod Rheumatol 2016;26:672-5.

27. Zhang S, Li L, Shen A, Chen Y, Qi Z. Rational use of tocilizumab in the treatment of novel coronavirus pneumonia. Clin Drug Invest 2020:1-8. https://doi.org/10.1007/s40261-020-00917-3.

28. The U.S. Food and Drug Administration. Information for ACTEMRA $®$ (tocilizumab). https://www.accessdata.fda.gov/drugsatfda_docs/ label/2017/125276s114lbl.pdf. [Accessed 15 Feb 2020].

29. Papp E, Mohammadi H, Loutfy MR, Yudin MH, Murphy KE, Walmsley SL, et al. HIV protease inhibitor use during pregnancy is associated with decreased progesterone levels, suggesting a potential mechanism contributing to fetal growth restriction. J Infect Dis 2015;211:10-8.

30. Roberts SS, Martinez M, Covington DL, Rode RA, Pasley MV, Woodward WC. Lopinavir/Ritonavir in pregnancy. J Acquir Immune Defic Syndr 2009;51:456-61.

31. Kawamura S, Russel AW, Freeman SJ, Siddal RA. [Reproduction and developmental study of meropenem in rats]. Nippon Kagaku Ryoho Gakkai Zasshi 1992;40(Suppl 1):238-50.

32. Bennett PN. Use of the monographs on drugs. In: Bennett PN, editor Drugs and human lactation, 2nd ed. London, UK: Elsevier; 1996.

33. Sperber K, Hom C, Chao CP, Shapiro D, Ash J. Systematic review of hydroxychloroquine use in pregnant patients with autoimmune diseases. Pediatr Rheumatol Online J 2009;7:9.

34. Oumar AA, Bagayoko-Maiga K, Bahachimi A, Maiga M, Cere MC, Diarra Z, et al. Efavirenz and lopinavir levels in HIV-infected women and their nursing infants, in Mali. J Pharmacol Exp Therapeut 2018;366:479-84.

35. Palombi L, Pirillo MF, Andreotti M, Liotta G, Erba F, Sagno JB, et al. Antiretroviral prophylaxis for breastfeeding transmission in Malawi: drug concentrations, virological efficacy and safety. Antivir Ther 2012;17:1511-9.

36. Corbett AH, Kayira D, White NR, Davis NL, Courti AP, Chasela C, et al. Antiretroviral pharmacokinetics in mothers and breastfeeding infants from 6 to 24 weeks post-partum: results of the BAN Study. Antivir Ther 2014;19:587-95. 